\title{
Lageabschätzung für einen Kondensator minimaler Kapazität -
}

\section{S. KIRSCH}

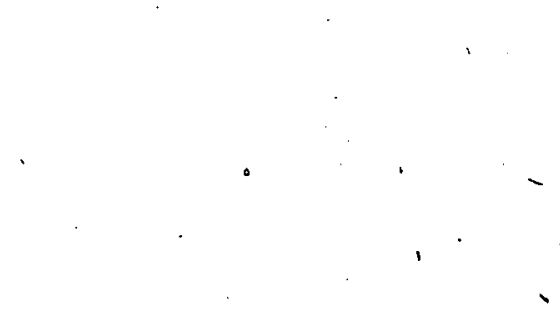

Mit Hilfe der Methode der inneren Variation werden die geometrische Gestalt und Lage von demjenigen Kontinuum untersucht, das $n \geqq 2$ fest und verschieden vorgegebene Punkte enthält und dessen verallgemeinerter transfiniter Durchmesser im Zusammenhang mit einer Fundamentallösung für ein verallgemeinertes Cauchy-Riemannsches, Differentialgleichungs. system minimal ausfällt.

С помощью метода внутренноп̆ вариации исследуются геометри́ческий вил и геометрическое положение континуума, содержащего $n \geqq 2$ различных фиксированных точек плоскости наименьшего обобщеңного диаметра, в связи с фундаментальным решением обобщенной системы дифференциальных уравнений Коши-Римана.

With the help of the method of interior variation we get informations about the geometrical form and locus from that continuum, which contains $n \geq 2$ various points and has minimal generalized transfinite diameter connected with a fundamental solution for generalized Cauchy-Riemann equations.

\section{Einleitung}

1968 behandelte R. KüHNaU [7] ein Extremalproblem, das, als ebenes elektrostatisches Problem gedeutet, in physikalischer Sprechweise folgendermaßen lautet. Zunächst heiße ein zweifach zusammenhängendes Gebict zulässiger Kondensator, wenn dessen Komplement aus dem unendlich fernen Punkt und einem Kontinuum $\mathfrak{R}$ besteht, das $n \geqq 2$ fest und verschieden vorgegebene Punkte $z_{1}, z_{2}, \ldots, z_{n}$ enthält. 'Zu charakterisieren ist die geometrische Gestalt und Lage der in einem inhomogenen Medium mit der Dielektrizitätskonstante $p(z), 1 / Q \leqq p(z) \leqq Q$, eingelagerten zulässigen Kondensatoren minimaler reduzierter Kapazität der Ladung 1 mit einer Gegenladung im unendlich fernen Punkt.

Hier ist folgendes Prinzip erfüllt. Es gibt genau einen zulässigen Kondensator minimaler reduzierter Kapazität. Das zugehörige, aus endlich vielen Jordänkurvenbögen bestehende Extremalkontinuum $\mathfrak{\Omega}^{\prime}$ besitzt die Eigenschaft $(E)$, da $B$ in jedem Punkt von $\mathfrak{N}^{\prime}$ die sich einstellende Ladungsdichte auf beiden Ufern iibereinstimmt.

Im Fall $p(z) \equiv 1$ handelt es sich um ein bekanntes, auf Tschebotarëw und Pólya zuriickgehendes klassisches Problem, das 1930 von H. Grötzscí [4] mit der Flächenstreifenmethode und der Kontinuitätsmethode dahingehend gelöst wurde, da $B$ das Extremalkontinuum aus einem höchstens $(2 n-3)$-fach verästelten analytischen Schlitz mit der Eigenschaft (E) besteht; vgl. [15] und dort genannte Iiteratur. Einfache a-priori-Schranken für die Lage des Extremalkontinuums im Fall $p \equiv 1$ sind aus der Literatur bekannt; z. B. $[8,18]$, wonach dieses ganz in der abgeschlossenen konvexen Hülle der Punkte $z_{1}, \ldots, z_{n}$ liegt.

In [7] wurde für $p(z) \neq 1$ die Extremalität und eindeutige Bestimmtheit des Extremalkontinuums mit der Eigenschaft (E) mittels Flächenstreifenmethode be- 
wiesen, während scine Existenz nur für den Fall $n=2$ durch Konstruktion einer gewissen Riemannschen Mannigfaltigkeit sichergestellt wurde. Das Hauptanliegen dieser Mitteilung besteht einerseits darin, die Existenz, des Extremalkontinuums für den Fall $n>2$ und ortsabhängiger Dielektrizitätskonstante $p(z)$ zu erbringen und andererseits eine a-priori-Schranke für dic Lage des Extremalkontinuums über seine Eigenschaft (E) abzuleiten. Fïr den Existenzbeweis eines Extremalkontinuums mit der Eigenschaft' $(\mathrm{E})$ wird allerdings vorausgesetzt, da $B$ in einer gewissen Uingebung des Extremalkontinuums $p(z) \equiv 1$ ist. Auch ohne diese Voraussetzung gilt die im. Satz 1 angejgebene Lageeinschränkung bei Kenntnis der Existenz des Extremalkontinuums mit der Eigenschaft (E) wie im Fall $n=2$.

\section{Bezeichnungen, Definitionen und Hilfssätze}

Mit $p(z)$ bezeichnen wir eine in der gesaniten $z$-Ebene $\mathbf{C}$ definierte reéllwertige und glatte Funktion bis auf eine Ausnahmemenge $S$, die aus endlich vielen analytischen Jordankurvenbögen bestehen möge, mit

$$
1 / Q \leqq p(z) \leqq Q,
$$

die in einer gewissen Umgebung des unendlich fernen Punktes identisch 1. sei. Wir setzen noch

$$
v(z)=\frac{p(z)-1}{p(z)+1}, \quad|\nu(z)| \leqq \frac{Q-1}{Q+1}<1 .
$$

$T(v)$ sei der Träger von $v(z)$, mit $D(\mathbb{N})$ bzw. $|\mathbb{N}|$ bezeichnen wir den Durchmesser bzw. den äußeren Jordanschen Flächeninhalt des Kontinuums $\mathfrak{R}$. Von den höchstens abzählbar unendlich vielen Gebieten, die $\mathbf{C} \backslash \mathfrak{R}$ ausmachen, bezeichnen wir dasjenige mit $G$, das den unendlich fernen Punkt im Inneren enthält.

Wir betrachten weiterhin die Klasse $\mathfrak{A}$ aller quasikonformen Abbildungen der Vollebene auf sich, deren Dilatation kleiner oder gleich $Q$ und identisch 1 in $\mathbf{C} \backslash T^{\prime}(\nu)$ ist und die in einer Umgebung des unendlich fernen Punktes, wo zwangsmäBig Konformität vorliegt, gemä $B z+a_{1} z^{-1}+, \cdots$ normiert sind.

In der Klasse $\mathfrak{A}$ gelten nach [11] für $z \in \mathbf{C}, z_{1}^{\prime}, z_{2} \in \mathbf{C} \backslash T(\nu)$ die Verzerrungsungleichungen

$$
\begin{aligned}
& |w(z)-z| \leqq \min \left\{\frac{1}{2 \pi}(Q-1)|T(v)| d^{-1}(z),(Q-1)(|T(v)| / \pi)^{1 / 2}\right\} \\
& \left|\log \frac{w\left(z_{1}\right)-w\left(z_{2}\right)}{z_{1}-z_{2}}\right| \leqq \frac{1}{2 \pi}(Q-1)|T(v)| d^{-1}\left(z_{1}, z_{2}\right) \\
& \left|w^{\prime \prime}\left(z_{1}\right) / w^{\prime}\left(z_{1}\right)\right| \leqq \frac{1}{2 \pi}(Q-1)|T(v)| d^{-3}\left(z_{1}\right)
\end{aligned}
$$

mit

$$
\begin{aligned}
& d(z)=\min \{|z-\zeta|: \zeta \in T(\nu)\} \quad \text { und } \quad d\left(z_{1}, z_{2}\right)=\min \left\{\left|z_{1}-\zeta\right| \cdot\left|z_{2}-\zeta\right|:\right. \\
& \zeta \in T(\nu)\} \text {. }
\end{aligned}
$$

Mit $w(z)$ bezeichnen wir die eindeutig bestimmte stetige schlichte Abbildung des einfach zusammenhängenden Gebietes $G$ auf das ÄuBere eines zu Null konzentrischen Kreises mit dem Radius $R(G, p)$, die $w(\infty)=\infty$ und für die log $w(z)$ die Differentialgleichung

$$
f_{\bar{z}}=\nu(z) \overrightarrow{f_{z}}
$$


erfüllt; wobei in $z=\infty$ die Entwicklung

$$
w(z)=z+\alpha_{0}+\alpha_{1} \cdot z^{-1}+\cdots
$$

, vorliegt (vgl. [17]).

Définition 1: $R(G, p)$ heißt quasikonformer Abbildungsradius von $G$.

Weiter bezeichnen wir mit $\mathfrak{r}(z, \zeta)$ die eindeutig bestimmte stetige schlichte $\mathrm{Ab}$ bildung der/Vollebene äuf sich mit $\mathfrak{r}(\zeta, \zeta)=0$ und $\dot{\mathfrak{r}}(\infty, \zeta)=\infty$, wobei $\log \mathfrak{r}(z, \zeta)$ fuir $z \neq \zeta$ die Differentialgleichung (4) erfüllt und in $z=\infty$ die Entwicklung

$$
\log \mathfrak{r}(z, \zeta)=\log z+\varepsilon^{\prime}(z, \zeta)
$$

bzw. in $z=\zeta \notin S$ die Entwicklung

$$
\log \mathfrak{r}(z, \zeta)=(1-v(\zeta))^{-1}(\log (z-\zeta)+v(\zeta) \cdot \overline{\log (z-\zeta)})+c(\zeta)+\varepsilon(z, \zeta)
$$

aufweist mit, nach Null strebenden Funktionen $\varepsilon^{\prime}(z, \zeta)$ (für $z \rightarrow \infty$ ) und $\varepsilon(z, \zeta)$ (für $z \rightarrow \zeta$ ) (vgl. [10, 21]).

Wir setzen noch

$$
[z, \zeta]=|\mathrm{r}(z, \zeta)| \text {. }
$$

Diese Abstandsfunktion ist symmetrisch und stetig in $z$ und $\dot{\zeta}$ (vgl. [12]). Für $p(z) \equiv 1$ ist $[z, \zeta] \equiv|z-\zeta|$ und für im Innern und Äußeren des Einheitskreises stückweise konstantes $p(z)$ ist $[z, \zeta]$ in [12] angegeben worden.

- Definition 2: Unter der $p$-Kapazität von $\mathfrak{R}$ verstehen wir die nichtnegative reelle Zahl'

mit

$$
\operatorname{cap}(\Re, p)=\exp (-V)
$$

$$
V=\inf (\mu, \mu), \quad(\mu, \mu) \stackrel{\vdots}{=}=-\iint \log [z, \zeta] d \mu(z) d \mu(\zeta)
$$

wobei dás Infimum über dèr Menge $\mathfrak{M}^{+}(\mathfrak{N})$ aller positiven $\sigma$-additiven Ladungen (= Mèngenfunktiónen), die auf' $\Re$ eingeschränkt sind und die Gesamitladung $\mu(\Re)=1$ aufweisen, genommen wird.

Da das Funktional $(\mu, \mu)$ nach unten halbstetig ist bezüglich dér schwachen Konvergenz, auf $\mathfrak{M}^{+}(\Re) \times \mathfrak{M}^{+}(\mathfrak{R})$, existiert eine sogenannte Gleichgewichtsladung $\mu \in \mathfrak{N} \mathfrak{l}^{+}(\mathfrak{N})$, für die $V=(\mu, \mu)$ gilt.

Wir setzen weiter

$$
d_{n}=\max \prod_{i<j}\left[z_{i}, z_{j}\right]^{1 /\left(\frac{n}{2}\right)} \quad \text { für . } z_{i} \in \mathfrak{N} \quad(i=1, \ldots, n ; n \geqq 2) .
$$

Wie im klassischen kọformen Fall $(p(z) \equiv 1)$ kann man zeigen (vgl. [6, 22]), daß $d_{n}$ mit wachsendem $n$ nicht zunimmt.

Definition 3: $d(\mathfrak{R}, p)=\lim d_{n}$ heißt verallgemeinerter transfiniter Durchmesser von $\Re$.

Für unsere weiterèn .Uberlegungen benötigen wir für die Abstandsfunktion $[z, \zeta]$ eine Verzerrungsaussage, die wir gleich allgemeiner für Abbildunge aus $\mathfrak{A}$ formulieren werden:

Mit der Hilfsfunktion gilt das

$$
H(x, y, t)=\left(x+2 y+2\left(x y+y^{2}\right)^{1 / 2}\right)^{1-1 / t}
$$


Lemma 1: Für $f(z) \in \mathfrak{A}$ und $\dot{z}, \zeta \in \mathbf{C}$ gilt

$$
B^{\prime}|z-\zeta|^{Q} \leqq|f(z)-f(\zeta)| \leqq A^{\prime}|z-\zeta|^{1 / Q}
$$

mit

$$
A^{\prime}=H(|z-\zeta|, D(T(\nu)), Q)
$$

und

$$
B^{\prime}=H\left(|z-\zeta|^{1 / Q} A^{\prime},\left(3+8^{1 / 2}\right)^{1-1 / Q} D(T(v)), Q\right)^{-Q} .
$$

-Bemerkung $1 \vdots(5)$ ist asymiptotisch scharf in dem Sinne, daß die linke und rechte Seite von (5) nach $|z-\zeta|$ streben für $Q \rightarrow 1$ bei festen $z, \zeta \in \mathbf{C}$.

Folgerung, 1: Wenn $|z-\zeta| \leqq D$ ist, so gilt für $f(z) \in \mathfrak{A}$

$$
B|z-\zeta|^{Q} Q \leqq|f(z)-f(\zeta)| \leqq A|z-\zeta|^{1 / Q}
$$

mit

und

$$
A=H(D, D(T(v)), Q) \leqq\left(2(D+2 D(T(v)))^{1-1 / Q}\right.
$$

$$
\begin{aligned}
B & =H\left(A D^{1 / Q},\left(3+8^{1 / 2}\right)^{1-1 / Q} D\left(T^{\prime}(v)\right), Q\right)^{-Q} \\
& \geqq\left[2\left(D^{1 / Q}\left(2\left(D+2 D\left(T^{\prime}(v)\right)\right)^{1-1 / Q}+2\left(3+8^{1 / 2}\right)^{1-1 / Q} D\left(T^{\prime}(v)\right)\right)\right)\right]^{1-Q} .
\end{aligned}
$$

Beweis zu Lemma 1: Sei $\Gamma$ die die endlichen und verschiedenen Punkte $z$ und $\zeta$ der $z^{\prime}$-Ebene verbindende. Strecke. Wir verwenden eine Aussage von A. Pfloger (vgl..[14: S. 49]) über die Veränderung des transfiniten Durchmessers von, $\Gamma$ gegenüber einem quasikonformen Homöomorphismus $f(z) \in \mathfrak{A}$, derzufolge

$$
d(f(\Gamma), 1) \leqq d(\Gamma, 1) \cdot \exp \int_{0}^{\infty}\left(1-1 / K_{\lambda}\right) d \lambda
$$

gilt. Dabei ist $K_{i}=\max _{z \in \Gamma_{\lambda}} \frac{\left|f_{z}\right|+\left|f_{z}\right|}{\left|f_{\bar{z}}\right|-\left|f_{\bar{z}}\right|} \leqq Q$ und $\Gamma_{\lambda}$ dieNiveaulinie $g\left(z^{\prime}, \infty\right)=\lambda(\mathbf{z u} z$ und $\zeta$ konfokale Ellipse) der Greenschen Funktion $g\left(z^{\prime}, \infty\right)$ des Äuß̉eren von $\Gamma$, die mit der Riemannschen Abbildungsfunktion $F\left(z^{\prime}\right)$ für das Äußere von $\Gamma$ durch $g\left(z^{\prime}, \infty\right)=\log \left|F\left(z^{\prime}\right)\right|$ zusammenhängt, wobei $F\left(z^{\prime}\right)=\left(l\left(z^{\prime}\right) / 2\right)+\left(\left(l\left(z^{\prime}\right) / 2\right)^{2}-1\right)^{1 / 2}$ mit $l\left(z^{\prime}\right)=\left(4 z^{\prime} /(\zeta-z)\right)+2((z+\zeta) /(z-\zeta))$ gilt.

Die rechte Seite von (7) weiter abgeschätzt liefert

$$
d(f(\Gamma), 1) \leqq d(\Gamma, 1) \cdot \exp \left((1-1 / Q)\left(\lambda_{1}-\lambda_{2}\right)\right)=d(\Gamma, 1)\left(r_{2} / r_{1}\right)^{1-1 / Q}
$$

wobei die reellen Zahlen $\lambda_{i}, \lambda_{2}>\lambda_{1} \geqq 0$, derart sind, da $\beta$ die konfokalen Ellipsen $\Gamma_{\lambda_{1}}, \Gamma_{\lambda_{1}}$, die den zu Null konzentrischen Kreisen mit den Radien $r_{1}$ und $r_{2}$ in der $F\left(z^{\prime}\right)$-Ebene entsprechen, den Träger $T(v)$ einschließen und berühren. Falls $T(v)$ und $\Gamma$ gemeinsame Punkte haben, setat man $\Gamma_{\lambda_{1}}=\Gamma$.

. Die große Halbachse von $\Gamma_{\lambda_{i}}$ sei $a_{i}=|z-\zeta|\left(r_{i}+1 / r_{i}\right) / 4(i=1,2) \mathrm{mit}$

und

$$
2 a_{1}=\left|z-z_{1}\right|+\left|\zeta-z_{1}\right|=\min _{z^{\prime} \in T(\nu)}\left(\left|z^{\prime}-z^{\prime}\right|+\left|\zeta-z^{\prime}\right|\right)
$$

$$
2 a_{2}=\left|z-z_{2}\right|+\left|\zeta-z_{2}\right|=\max _{z^{\prime} \in T(v)^{\prime}}\left(\left|z-z^{\prime}\right|+\left|\zeta-z^{\prime}\right|\right)
$$


Wird im Zähler des Ausdruckes

$$
\frac{r_{2}}{r_{1}}=\frac{\left(\left|z-z_{2}\right|+\left|\zeta-z_{2}\right|\right)+\left[\left(\left|z-z_{2}\right|+\left|\zeta-z_{2}\right|\right)^{2}-|z-\zeta|^{2}\right]^{1 / 2}}{\left(\left|z-z_{1}\right|+\left|\zeta-z_{1}\right|\right)+\left[\left(\left|z-z_{1}\right|+\left|\zeta-z_{1}\right|\right)^{2}-|z-\zeta|^{2}\right]^{1 / 2}}
$$

jeweils der Summand in runden Klammern durch $\left|z-z_{1}\right|+\left|z_{1}-\zeta\right|+2 D(T(v))$ vergrößert, so entsteht ein Ausdruck, der als Funktion von $\left|z-z_{1}\right|+\left|z_{1}-\zeta\right|$ $(\geqq|z-\zeta|$ ) monoton fallend ist. Es gilt dann

$$
\frac{r_{2}}{r_{1}} \leqq \frac{|z-\zeta|+2 D(T(v))+\left[\left(|z-\zeta|+2 D(T(v))^{2}-|z-\zeta|^{2}\right)\right]^{1 / 2}}{|z-\zeta|} .
$$

$\dot{V}$ erbindet man (8) und (9) mit dèr bekannten Ungleichung zwischën dem Durchmesser und dem gewöhnlichen transfiniten Durchmesser von $f(\Gamma)$ :

$$
D(f(\Gamma)) \leqq 4 d(f(\Gamma), 1)
$$

.so erhält man unter Berücksichtigung von $d(\Gamma, 1)=|z-\zeta| / 4$ die rechte Seite von (5) und zuglcich die Abschätzung

$$
D(f(T(v))) \leqq\left(3+8^{1 / 2}\right)^{1-1 / Q} D(T(v)) .
$$

Da die Umkehrfunktion $z=z(f)$ von $f=f(z) \in \dot{\mathfrak{A}}$ ebenfalls eine $Q$-quasikonforme Abbildung der Vollebene auf sich ist mit $z(\infty)=\infty$ und $z^{\prime}(\infty)=1$, so erhält man nach Anwendung der rechten Seite von (5) auf $z=z(f)$ die linke Seite der Ungleichung (5)

Lemma 2: Sei $G_{n} \ni \infty$ eine Folge einjach zusammenhïngender Gebiete, die das einfach, zusammenhängende Gebiet $G \ni \infty$ als Kern im Sinne von [3: S. 196] besitzt und gegen ihn konvergiert. Des weiteren sei $w_{n}(z)$ bzw. $w(z)$ die zugehörige quasikonforme . Kreisabbildung von $G_{n}$ bzw. G. Dann konvergieren die. Abbildungsfunktionen $w_{n}(z)$ im Innern von $G$ gleichmäßig gegen die Abbildungsfinktion $w(z)$, die Bildgebiete $w_{n}\left(G_{n}\right)$ besitzen $w(G)$ als Kern und konvergieren gegen ihn, d. $h . R\left(G_{n}, p\right) \rightarrow R(G, p)$ für $n \rightarrow \infty$. ।

Es sei darauf hingewiesen, daß es, obwohl die Abbildungsfunktionen $w_{n}(z)$ in gewissen Punkten von $G$ erst'von einem gewissen $n$ ab erklärt sind, dennoch sinnvoll ist, von Konvergenz in diesen Punkten zu reden.

Beweis: Die Funktionenfolge $w_{n}(z)$ ist in $G$ punktweise konvergent. Andernfalls gäbe es zwei Teilfolgen aus $w_{n}(z)$, die in einem Punkt von $G$ gegen zwei verschiedene Grenzwerte' ḱonvergieren würden. Aus diesen zwei Teilfolgen kann man aus Normalitätsgründen wieder je eine Teilfolge $w_{n}^{(1)}(z)$ bzw. $w_{n}^{\left({ }^{2}\right)}(z)$ so auswählen, daß sie im Inneren von $G$ gleichmäßig gegen die quasikonforme Abbildungsfunktion $w^{(1)}(z)$ bzw. $w^{(2)}(z)$ mit

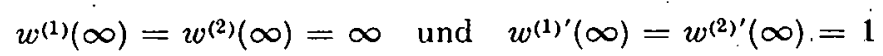

ḱnvergiert und daß die Bildgebietsfolge ${ }^{-} w_{n}^{(1)}\left(G_{n}\right)$ bzw. $w_{n}{ }^{(2)}\left(G_{n}\right)$ kernkonvergent gegen das Kreisgebiet $|w|>R_{1}$ bzw. $|w|>R_{2}$ ist. In Verbindung mit einem Kernkonvergenzsatz für quasikonforme Abbildungen [16: S. 79] folgert man, daß die quasikonforme Grenzabbildung $w^{(i)}(z)$ das Gebiet $G$ auf $|w|>R_{i}(i=1,2)$ abbildet. - $\mathrm{Da}$ in $G \backslash S$

$$
\lim _{n \rightarrow \infty} w_{n_{\bar{z}}}^{(i)} / \overline{w_{n_{\Sigma}}^{(i)}}=\lim _{n \rightarrow \infty} v(z) w_{n}^{(i)}(z) / \overline{w_{n}^{(i)}(z)}=v(z) w^{(i)}(z) / \overline{w^{(i)}(z)}
$$

für $i=1,2$ gilt, stimmt die komplexe Dilatation zweiter Art von $w^{(i)}(z)$ in $G$ mit $v(z) w^{(i)}(z) / \widehat{w^{(i)}(z)}$ bis auf eine Menge vom Lebesgues̉chen Maß Null iiberein (vgl. [16: Satz 5.2, S. 197]), wenn man dort nur $w_{z}$ und $w_{n_{z}}$ durch ihré konjugiert kom- 
plexen Werte ersetzt. Wegen der eindeutigen. Bestimmtheit von $\dot{w}(z)$ folgt $w^{(1)}(z)$ $\equiv w^{(2)}(z)$ in $G$, im Widerspruch zur Annahme. Insbesondere folgt aus den Úberlegungen die Kernkonvergenz von $w_{n}\left(G_{n}\right)$ gegen ein Kreisgebiet $|w|>R$. Die Punktkonvergenz in' Verbindung mit der Normalität der Folge $w_{n}(z)$ liefert die gleichmäßige Konvergenz im Innern von $G$ gegen eine quasikonforme Abbildungsfunktion $w^{*}(z)$, die $G$ auf $|w|>R$ abbildet und deren Logarithmus fast überall in $G$ die Differentialgleichung (4) erfüllt. Wegen der eindeutigen Bestimmtheit von $w(z)$ gilt $w(z) \equiv w^{*}(z)$ in $G$ und $R\left(G_{n}, p\right) \rightarrow R=R(G, p)$ für $n \rightarrow \infty$

Bemerkung 1: Das Lemma 2 gilt sinngemä $B$ auch für den Fall, wenn $G_{n} \equiv G$ gesetzt wird, und $w_{n}(z)$ die quasikonforme Kreisabbildungsfunktion von $\dot{G}$ mit der komplexen Dilatation zweiter Art $\nu_{n}(z) w_{n}(z) / \overline{w_{n}(z)}$ bedeutet, wobei $v_{n}(z)$ in der gesamten Ebene erklärte, reellwertige und zweimal stetig differenzierbare Funktionen mit kompaktem Träger bezeichnen; die fast überall gegen $v(z)$ konvergieren.

L'émma $3:$ Es gilt

und

$$
d(\mathfrak{R}, p)=\dot{\operatorname{cap}}(\mathfrak{\Re}, p)=R(\dot{G}, p)
$$

$$
B R(G, 1)^{Q} \leqq R(G, p) \leqq A R(G, 1)^{1 / Q}
$$

mit Konstanten $A, B$ wie in (6), wenn dort $\bar{D}=D(\mathfrak{I})$ gesetat wird.

Beweis: Zum Beweis der ersten Gleichung in (11) vergleiche man [6] und [22]. Sei $G_{n}$ eine $G$ ausschöpfende' monotone Folge einfách zusammenhängender Gebiete, die analytisch berandet seien. Mit $\mu$ bzw. $\mu_{n}$ bezeichnen wir die Gleichgewichtsladungen für die Kontinuen $\mathfrak{R}$ bzw. $\mathfrak{R}_{n}$. Aus der Definition 2 folgert man

$$
\left(\mu_{n}, \mu_{n}\right) \leqq\left(\mu_{n+1}, \mu_{n+1}\right) \leqq(\mu, \mu),
$$

so $\operatorname{da} B \lim _{n \rightarrow \infty}\left(\mu_{n}, \mu_{n}\right) \leqq(\mu, \mu)$ ist. Wegen der schwachen Kompaktheit der Folge $\mu_{n} \in \mathfrak{M}^{+}\left(\Re_{n}\right)$ existiert eine Teilfolge von $\mu_{n}$, wieder mit $\mu_{n}$ bezeichnet, die schwach gegen ein $\mathrm{Ma}^{\prime} \mu^{\prime} \in \mathfrak{M}^{+}(\mathfrak{N})$. konvergiert. Wegen der Halbstetigkeit nach unten des Funktionals $(\mu, \mu)$ auf $\mathfrak{M}^{+}(\mathfrak{N}) \times \mathfrak{M}^{+}(\mathfrak{N})$ erhält man somit unter Berücksichtigung von Definition 2

$$
(\mu, \mu) \leqq\left(\mu^{\prime}, \mu^{\prime}\right) \leqq \lim _{n \rightarrow \infty}\left(\mu_{n}, \mu_{n}\right) \leqq(\mu, \mu) ; .
$$

woraus $\operatorname{cap}(\Re, p)=\lim _{n \rightarrow \infty} \operatorname{cap}\left(\Re_{n}, p\right)$ folgt. Nach [6: Satz 2] gilt-nun cap $\left(\Re_{n}, p\right)$ $=R\left(G_{n}, p\right)$. Alles zusammengefaßt liefert dies in Verbindung mit Lemma 2 die Gültigkeit der zweiten Gleichung von Lemma 3. Die Doppelungleichung ergibt sich ,aus (11), Definition 3 und (6), wobei in (6) $w(z)=\mathfrak{r}(\dot{z}, \zeta)$ gesetzt wurde

\section{'3. Aufgabenstellung und Hauptergebnis}

Unter allen Kontinuen $\mathfrak{R}$, die $n \geqq 2$ fest und verschieden vorgegebene Punkte $z_{1}, \dot{z}_{2}, \ldots, z_{n}$ enthalten, sind diejenigen Kontinuen $\widehat{\Omega}^{\prime}$ gesucht, für die der betreffende quasikonforme Abbildungsradius des Äußeren $G^{\prime}$ von $\mathfrak{H}^{\prime}$ minimal ausfällt. 


\subsection{Existenzbeweis von $\mathfrak{R}^{\prime}$}

$G_{1}$ sei das Åußere der abgeschlossenen konvexen Hülle $\Re_{1}$ der Punkte $\dot{z}_{1}, \ldots, z_{n}$. Aus der Menge der zulässigen Vergleichskontinuen, deren $\vec{A} u$ Beres $G$ einen quasikonformen Abbildungsradius

$$
R(G, p) \leqq R\left(G_{1}, p\right)
$$

aufweisen, sondern wir eine Extremalfolge $\mathfrak{t}_{m}=\mathrm{C} \backslash G_{m}$ mit $R\left(G_{m}, p\right) \searrow R^{4}=\min$ aus. Mit $z_{m}(w)$ bezeichnen wir die quasikonforme Umkehrabbildung von $w_{m}(z): G_{m} \rightarrow$, $|w|>R\left(G_{m}, p\right)$, die wegen (4) der Beltrami-Differentialgleichung

$$
\dot{z_{m_{\bar{w}}}}=-\frac{w}{\bar{w}} \nu\left(z_{m}(w)\right) z_{m_{\mathrm{w}}} \quad \text { in }|w|>R\left(G_{m}, p\right)
$$

genügt. Dên Ungleichungen (10) und (12) entnimmt man die gleichmäßige Beschränktheit der Extremalfolge $\Omega_{m}$. Aus Kompaktheitsgründen kann man aus der Funktionenfolge $z_{m}(w)$ eine Teilfolge, wieder mit $z_{m}(w)$ bezeichnet, auswählen, die im Innern von $|w|>R=$ min gleichmäßig gegen eine nicht konstante quasikonforme Abbildung $z(w)$ konvergiert. Wegen der Glattheitsvoraussetzung über $v(z)$ gilt bis auf diejenigen Punkte von $|w|>K$, wo $v(z(w))$ eventuell unstetig ist,

$$
\lim _{m \rightarrow \infty} z_{m \bar{w}} / z_{m w}=\lim _{m \rightarrow \infty}-v\left(z_{m}(w)\right) w / \bar{w}=-v(z(w)) w / \bar{w}
$$

d. h. $z_{m}(w)$ stellt eine gute Approximation von $z(w)$ im Sinne von [16: S. 197] dar, so $\operatorname{da} B z(w)$ f. ï. (13) in $|w|>R$ und damit $\log w(z)$ die Differentialgleichụng (4) f. ü. in $G^{\prime}=z(|w|>R)$ erfüllt. $\widehat{\Omega}^{\prime}=\mathbf{C} \backslash G^{\prime}$ ist offenbar ein die Punkte $z_{1}, \ldots, z_{n}$ enthaltendes Kontinuum, für dessen Äußeres der quasikonforme Abbildungsradius minimal ausfällt.

\subsection{Eine grobe Lageabschätzung für $\mathfrak{R}^{\prime}$}

Aus (10), (12) und der Extremalität von $\mathfrak{K}^{\prime}$ erhalten wir für den Durchmesser von $\mathfrak{R}^{\prime}$ die Abschätzung

$$
\begin{aligned}
D\left(\AA^{\prime}\right) \leqq & 4^{Q} D^{1 / Q}\left(2\left(D+2 D\left(T^{\prime}(\nu)\right)\right)\right)^{1-1 / Q} \\
& \times\left(2\left(1+2 D(T(\nu)) D^{-1}\right)^{1-1 / Q}+2\left(3+8^{1 / 2}\right)^{1-1 / Q} D(T(\nu)) D^{-1}\right)^{Q-1}=: r^{\prime}
\end{aligned}
$$

wobei $D=\max _{i \neq j}\left|z_{i}-z_{j}\right|$ gesetzt ist: Daraus schließt man, daß $\mathfrak{R}^{\prime}$ ganz in dem Durchschnitt $M \stackrel{i \phi j}{=} M\left(z_{1}, \ldots, z_{n}, r^{\prime}\right)$ der abgeschlossenen. Kreisscheiben $\left|z-z_{i}\right| \leqq$ ?' $(i=1, \ldots, n)$ liegt. Im weiteren setzen wir voraus, $\mathrm{d} a B \mathfrak{R}^{\prime}$ und $T(\nu)$ keine gemeinsamen Punkte besitzen. Das ist sicher dann gẹährleistet, wenn der Abstand

$$
l=\min _{z \in M, \varepsilon \in T(\nu)}|z-\zeta|
$$

des Trägers von $v(z)$ und $M$ größer Null ist. 
3.3. Variationsformel für den quasikonformen Abbildungsradius $\boldsymbol{R}(\boldsymbol{G}, \boldsymbol{p})$, falls $z_{i} \notin G \supset T(v), i=1, \ldots, n$

Wir setzen zunächst $G$ analytisch berandet voraus. Die reelle Funktion $v(z)$ sei vorerst zweimal stetig differenzierbar, wobei der kompakte Träger $T(v) \subset G$ den Rand von $G$ vom unendlich fernen Punkt nicht trennen möge. Von diesen Glattheitsvoraussetzungen werden wir uns später lösen.

Sei $C$ eine beliebige, -aber feste, $T(v)$ umschlingende geschlośsene analytische Jordankurve innerhalb von $G$. Des weiteren bezeichnen wir nit $k_{2}$ einen Kreis mit dem Mittelpunkt $z$ und Radius $\varrho$ im Äußeren von $\partial G$ und $C$. Die Kurven $\partial G, C$ und $k_{z}$ seien mathematisch positiv orientiert. Die innere Variation

$$
\zeta_{\varepsilon}=\zeta+\varepsilon(\zeta-z)^{1-n} \prod_{i=1}^{n}\left(\zeta-z_{i}\right), \varepsilon \text { komplexe Konstanté, }
$$

vermittelt aú ßerhalb von $k_{z}$ eine schlicht konforme Abbildung für hinreichend kleines $|\varepsilon|$.

Wir setzen noch

$$
P(\zeta)=\log (w(\zeta) / R(G, p)), \quad . P_{\varepsilon}(\zeta)=\log \left(w_{\varepsilon}(\zeta) / R\left(G_{\varepsilon}, p\right)\right),
$$

wenn $w(\zeta)$ bzw. $w_{\varepsilon}(\zeta)$ die zugehörige quasikonforme Kreisabbildung von $G$ bzw. $G_{c}$ $\left(=A u\right.$ Beres von $\left.\zeta_{s}(\partial G)\right)$ bedeutet. Nach dem Cauchyschen Integralsatz gilt

$$
\begin{aligned}
\operatorname{Re} \frac{1}{2 \pi i} \cdot \int_{\partial G+C+k_{\varepsilon}}\left[P_{\varepsilon}\left(\zeta_{\varepsilon}\right)-P(\zeta)\right] d P= & \log \left(R(G, p) / R\left(G_{\varepsilon}, p\right)\right) \\
& +\operatorname{Re} \log (1+\varepsilon) .
\end{aligned}
$$

Da $P(\zeta)$ und $P_{\varepsilon}(\zeta)$ Lösungen von (4) sind, gilt für hinreichend kleines $|\varepsilon|$

$$
\operatorname{Re} \frac{i}{2 \pi i} \int_{C+k_{i}}\left[P(\zeta)-P_{c}(\zeta)\right] d P=0
$$

Die Gleichungen (15) und (16), addiert, liefern uns unter Berücksichtigung von

$$
\operatorname{Re} \frac{1}{2 \pi i} \int_{\partial G}\left[P_{\imath}\left(\zeta_{\varepsilon}\right)-P(\zeta)\right] d P \stackrel{\vdots}{=} 0
$$

die Bezichung

$$
\begin{aligned}
\log \left(R(G, p) / R\left(G_{\varepsilon} ; p\right)\right)= & \operatorname{Re} \frac{1}{2 \pi i} \int_{C+k_{z}}\left[P_{\varepsilon}\left(\zeta_{\varepsilon}\right)-P_{\varepsilon}(\zeta)\right] P^{\prime}(\zeta) d \zeta \\
& -\operatorname{Re} \log (1+\varepsilon) .
\end{aligned}
$$

Durch eine Ausschöpfung von $G$ durch analytisch berandete Gebiete schließen wir in Verbindung mit Lemma 2 auf die Gültigkeit von (18) für beliebig berandete Gebiete $G$. Approximiert man $v(z)$ mit $T^{\prime}(v) \subset G$ durch eine Folge von zweimal stetig differenzierbaren Funktionen $v_{n}(z)$ mit kompakten Trägern $T^{\prime}\left(v_{n}\right) \subset G$, die $\partial G$ von $\infty$ nicht trennen mögen, so bleibt mit der Bemerkung 1 die Variationsformel (18) auch für den Fall einer stiickweise glatten Funktion $v(z)$ mit kompaktem Träger $T(\nu) \subset G$ bestehen. Falls $T^{\prime}(v)$ den Rand $\partial G$ von $\infty$ trennt, hat man vor dem letztgeñannten Grenzübergang in (18) die Kurve $C$ durch zwei geschlossene analytische Jordankurven $C_{1}$ (mathematisch negativ orientiert) und $C_{2}$ (mathematisch positiv 
* orientiert) zu ersetzen, so daß $\partial G$ im Innern von $C_{1}, C_{1}$ im Innern von $C_{2}$ und $T^{\prime}(v)$ zwischen $C_{1}$ und $C_{2}$ liegt.

$\mathrm{Da}$ in einer gewissen. Ungebung $U$ von $k_{z}+C$ für hinreichend kleines $|\varepsilon|$ die Entwicklung

$$
P_{\varepsilon}\left(\zeta_{\varepsilon}\right)-P_{\varepsilon}(\zeta)=P_{\varepsilon}^{\prime}(\zeta)\left(\zeta_{\varepsilon}-\zeta\right)+o(\varepsilon)
$$

und auf Grund von Lemma 2 .

$$
P_{\varepsilon}^{\prime}(\zeta)^{\prime}=P^{\prime}(\zeta)+h^{\prime}(\varepsilon, \zeta)
$$

gilt mit $h(\varepsilon, \zeta)$ gleichmäßig konvergent gegen Null in $U$ für $|\varepsilon| \rightarrow 0$, geht (18) nach Anwendung der Cauchyschen Integralformel über in

$$
\begin{aligned}
\log \left(R(G, p) / R\left(G_{\varepsilon}, p\right)\right)= & \operatorname{Re} \varepsilon\left[\frac{d^{n-2}}{d z^{n-2}}\left(\frac{P^{\prime 2}(z) \prod_{i=1}^{n}\left(z-z_{i}\right)}{(n-2) !}\right)\right. \\
& \left.+\frac{1}{2 \pi i} \int_{c} P^{\prime 2}(\zeta)(\zeta-z)^{1-n} \prod_{i=1}^{n}\left(\zeta-z_{i}\right) d \zeta-1\right]+o(\varepsilon)
\end{aligned}
$$

Unter der Voraussetzung positiven Abstandes $l$ zwischen $T(v)$ und $M$ gilt

' Satz 1. Es gibt genau ein Extremalkontinuum $\mathfrak{\Re}^{\prime}$, für dessen Äußeres $G$ ' der quasikonforme Abbildungsradius $R\left(G^{\prime}, p\right)$ minimal ausfällt. Es besteht aus einem höchstens $(2 n-3)$-fach verästelten analytischen Schlitz mit der Eigenschaft $(E), d . h$. da $\beta$ dem links- und rechtsseitigen Ujer eines beliebigen Teilbogens von $\mathfrak{K}^{\prime}$ auf dem Kreis $|w|$ $=R\left(G^{\prime}, \dot{p}\right)$ bei der. Extremalabbildung $u(z)$ gleich lange Kreisbögen entsprechen. Dus. Extremalkontinuum wird durch ein positives quadratisches Differential

$$
Q(z) d z^{2}>0, \quad Q(z) \text { meromorph in } \mathbf{C} \backslash C,
$$

beschrieben und liegt ganz in der abgeschlossenen konvexen Hülle der Vereinigung der Kreisscheiben

$$
\left|z-z_{i}\right| \leqq \min \left\{\pi^{-1}(Q-1)|T(\nu)| / l, 2(Q-1)\left(\left|T^{\prime}(\dot{\nu})\right| / \pi\right)^{1 / 2}\right\} .
$$

für $i=1, \ldots, n$.

- Sati 1'. 'Im. Fall $n=2$ ist das Extremalkontinuum $\Omega^{\prime}$ das analytische Bild einer Strecke, vermittelt durch eine quasikonforme Abbildung der Vollebene auf sich: Wenn $\alpha(z)$ bzw. $x(z)$ den Winkel der von $z_{1}$ nach $z_{2}$ gerichteten Tangente zur Richtung von $z_{1}$ nach $z_{2}$ bzw. die Krümmung des anulytischen Jordankurvenbogens $\mathfrak{N}^{\prime}$ mit der Länge $L$ im Punkt $z \in \mathbb{R}^{\prime}$ bezeichnet, so gelten noch zu der a priori Lageeinschränkung von $\mathfrak{T}^{\prime}$ im Salz 1 die folgenden Abschälzungen:

und

$$
\begin{aligned}
& |\alpha(z)| \leqq \pi^{-1}(Q-1)\left|T^{\prime}(\nu)\right| l^{-2}, . \quad z \in \mathfrak{I}^{\prime}, \\
& |\varkappa(z)| \leqq(2 \pi)^{-1}(Q-1)\left|T^{\prime}(\nu)\right| l^{-3}, z \in \mathfrak{I}^{\prime},
\end{aligned}
$$

$$
\begin{gathered}
\left|z_{1}-z_{2}\right| \leqq L \leqq \cdot\left|z_{1}-z_{2}\right|^{1 / Q}\left(2\left(\left|z_{1}-z_{2}\right|+2 D(T(v))\right)\right)^{1-1 / Q} \\
\quad \times \exp \left(\frac{1}{2 \pi} \cdot(Q-1)\left|T^{\prime}(\nu)\right| l^{-2}\right) .
\end{gathered}
$$

Bemerkung 2: Die Lageabschätzung des Extremalkontinuums $\boldsymbol{T}^{\prime}$ für den Fall $n=2$ im Șatz 1 gilt,übrigens auch für eine beliebige Lage des Trägers vọn $v(z)$, da 
die Eigenschaft $(E)$ von $\mathfrak{I}^{\prime}$ unabhängig von der Lage des Trägers von $v(z)$ in [7] über die Konstruktion einer gewissen Riemannschen Mannigfaltigkeit nachgewiesen wurde.

Bemerkung 3: Daß $\mathfrak{R}^{\prime}\left(z_{1}, z_{2}\right)$ im Fall $p(z) \geqq 1$ das Bild einer Strecke ist, vermittelt durch eine quasikonforme Abbildung der Vollebene auf sich, wurde bereits in [13: S. 245] bemerkt.

Beweis von Satz 1: Sei $G^{\prime}$ ein Extremalgebiet. $P(z)$, der Logärithmus der zugehörigen Extremalabbildung, erfüllt dann in $G^{\prime}$ rechterhand von $C$ notwendig die Differentialgleichung

$$
\begin{aligned}
P^{\prime 2}(z) \prod_{i=1}^{n}\left(z-z_{i}\right)= & \left(z^{n-2}+b_{n-3} z^{n-3}+\cdots+b_{0}\right) \\
\quad & -\frac{1}{2 \dot{\pi} i} \int_{c}^{\frac{P^{\prime 2}(\zeta) \prod_{i=1}^{n}\left(\zeta-z_{i}\right)}{\zeta-z^{\prime}} d \zeta,}
\end{aligned}
$$

$b_{i}$ konstant $(i=0, \ldots, n-3)$.

Dies ergibt sich durch $(n-2)$-malige Integration des eckigen Klammerausdruckes in (19), der identisch verschwinden muß. Offenbar kann $\mathfrak{R}^{\prime}$ keine inneren Punkte besitzen. Andernfalls könnte man $\mathfrak{\Re}^{\prime}$ stets so abändern, daß der quasikonforme Abbildungsradius $R(G, p)$ als streng monoton fallendes Gebietsfunktional (vgl. [6]) kleiner als $R\left(G^{\prime}, p\right)$ ausfallen würde im Widerspruch zur Minimalität von $R\left(G^{\prime}, p\right)$. Setzt man.

$$
f(z)=P^{\prime 2}(z) \prod_{i=1}^{n}\left(z-z_{i}\right)-\left(z^{n-2}+b_{n-3} z^{n-3}+\cdots+b_{0}\right)
$$

und wendet die Sochozki-Plemeljschen Formeln auf (23) an, so erkennt man, daß $f(z)$ die homogene Integralgleichung

$$
f(z)=-\frac{1}{\pi i} \int_{C} \frac{f(\zeta)}{\zeta-z} d \zeta, \quad z \in C
$$

- erfüllt.

Der Differentialgleichung (23) entnimmt man, da $B \Omega^{\prime}$ durch das positive quadratische Differential

$$
Q(z) d z^{2}>0
$$

mit

$$
Q(z) \fallingdotseq-\frac{z^{n-2}+b_{n-3} z^{n-3}+\cdots+b_{0}-(2 \pi i)^{-1} \int_{c} f(\zeta)(\zeta-z)^{-1} d \zeta}{\prod_{i=1}^{n}\left(z-z_{i}\right)}
$$

beschrieben wird. Nach [5] besteht $\mathfrak{\Re}^{\prime}$ deminach aus analytischen Schlitzen, die in höchstens endlich vielen Verzweigungspunkten (= Nullstellen des Zählers von $Q(z)$ ) verästelt sein können und in mindestens zwei der Punkte $z_{1}, \ldots, z_{n}$ einmünden müssen. I etzteres bedeutet; daß $Q(z)$ mindestens zwei einfache Pole besitzen muß, andernfalls wäre der topologische Verlauf des Extremalkontinuums unvereinbar mit der Extremalität von $\mathfrak{\Re}^{\prime}$. Die Eigenschaft $(E)$ von $\mathfrak{R}^{\prime}$ ergibt sich dann vermöge der 
Beziehung

$$
\left(\frac{\partial}{\partial n} \log |w(z)|\right)^{2}=-z^{\prime 2}(s) P^{\prime 2}(z),
$$

wobei $z(s)$ eine Parameterdarstellung von $\mathfrak{R}^{\prime}$ bezuiglich der Bogenlänge $s$ und $n$ die Außennormale bezeichnet, und unter Berücksichtigung der Nichtnegativität der Nórmalenableitung von $\log |w(z)|$ in $z \in \mathbb{R}^{\prime}$ in Verbindung mit (23). Mit $w_{i}=w\left(z_{i}\right)$ bezeichnen wir den Bildpunkt von $z_{i}$ (= einfacher Pol von $Q(z) ; i=1, \ldots, r \leqq n$ ) .bezüglich der Extremalabbildung $w(z)$ auf dem Kreis $|w|=R\left(G^{\prime}, p\right)$. Wir konstruieren durch Ränderzuordnung eine Riemannsche Mannigfaltigkeit, in dem wir jeweils, falls vorhanden, die $m$ Bildpunkte beziiglich der Extremalabbildung $w(z)$ auf dem Kreis $|w|^{\prime}=R\left(G^{\prime}, p\right)$ jedes $m$-fach erreichbaren Punktes von $\mathfrak{R}^{\prime}$ identifizieren. Insbesondere werden die $w_{i}$ sich selbst zugeordnet $(i=1, \ldots, r \leqq n)$. Nach èinem Fundamentalsatz der konformen Abbildung läßt sich die' so gefundene Riemannsche Mannigfaltigkeit, die von den Punkten $w_{i}(i=1, \ldots, r \leqq n)$ begrenzt zu denken ist und $\infty$ als inneren Punkt enthält, auf einen schlichten $r$-fach zusammenhängenden Bereich der $\zeta$-Ebene schlicht konform abbilden, so da $\beta \zeta(\infty)=\infty$ und $\zeta^{\prime}(\infty)=1$ gilt. Den Begrenzungen $w_{i}$ entsprechen notwendig die Punkte $\zeta_{i}(i=1, \ldots, r \leqq n)$. Die Kreislinie $|w|=R\left(G^{\prime}, p\right)$ geht in einen höchstens $(2 r-3)$-fach verästelten analytischen Schlitz $\mathfrak{\Omega}^{\prime \prime}$ über, der nach [4] gerade das eindeutig bestimmte, die Punkte $\zeta_{i}(i=1, \ldots, r \leqq n)$ enthaltende Extremalkontinuum fiur den konformen Fall $v(z) \equiv 0$ ist. (Auf die Möglichkeit, die eben angegebene konforme Verheftung von $|w|=R\left(G^{\prime}, p\right)$ auch nutzbar zu machen für eine a priori-Iageabschätzung von $\mathbb{R}^{\prime}$ für $n>2$, machte mich Herr R. Kühnau aufmerksam.) Die zusammengesetzte Abbildung

$$
\zeta=\zeta(w(z))
$$

mit $\zeta^{\prime}(\infty)=1$ ist eine die Punkte $z_{i}$ in $\zeta_{i}(i=1, \ldots, n)$ überführende $Q$-quasizonforme.Abbildung des Äußeren von $\mathfrak{R}^{\prime}$ auf das Äußere von $\mathfrak{I}^{\prime \prime}$, die auf Grund der obigen Verheftungsvorschrift zu einer topologischen Abbildung der Vollebene auf sich erweitert werden kann. Da eine $Q$-quasikonforme Abbildung bezüglich analytischer Bögen hebbar ist, gehört (24) nach hydrodynamischer Normierung in $z=\infty$ zu $\mathfrak{A}$. Wegen $\mathfrak{\mathbb { R }}^{\prime}=\zeta^{-1}\left(\mathfrak{I}^{\prime \prime}\right)$ ist $\mathfrak{\Re}^{\prime}$ höchstens $(2 n-3)$-fach verästelt. Bekanntlich liegt $\mathfrak{R}^{\prime \prime}$ ganz in dèr abgeschlossenen konvexen Hülle $H\left(\zeta_{1}, \ldots, \zeta_{n}\right)$ der Punkte $\zeta_{1}, \ldots, \zeta_{n}$ (vgl. [8]). Identifiziert man die $z$-Ebene und $\zeta$-Ebene, so entnimmit man (1), daß $\zeta_{i}=\zeta\left(z_{i}\right)$ innerhalb des Kreises

$$
\left|z-z_{i}\right| \leqq \min \left\{(Q-1)\left(\left|T^{\prime}(\nu)\right| / \pi\right)^{1 / 2}, \frac{1}{2 \pi}(Q-1)|T(\nu)| l^{-1}\right\},
$$

fiir $i=1, \ldots, n$, liegt. Daher liegt $H\left(\zeta_{1}, \ldots, \zeta_{n}\right)$ innerhalb der abgeschlossenen konvexen Hülle $H^{\prime}$ der Vereinigung der Kreise (25). Wendet man abermals (1) auf die Punkte $\zeta$ von $H^{\prime}$ an unter Beriicksichtigung der Schlichtheit von (24), so folgt die Behauptung des Satzes 1 über die L'age von $\mathfrak{R}^{\prime}$.

Die eindeutige Bestimmtheit von ' $\AA^{\prime}$ beweist man mit der Eigenschaft $(E)$ wie in [7]

Beweis von Satz 1': Im Fall $n=2$ ist $\mathfrak{I}^{\prime \prime}$ die $\zeta_{1}$ und $\zeta_{2}$ verbindende Strecke und damit $\mathfrak{I}^{\prime}$ das analytische Bild einer Strecke, vermittelt durch eine $Q$-quasikonforme - Abbildung der Vollebene auf sich. Sei $\zeta^{-1}(z)$ die Umkehrfunktion von (24). Fiir $\mathfrak{I}^{\prime}$ - wählen wir die Parameterdarstellung

$$
z(t)=\zeta^{-1}\left(\zeta_{1}+t\left(\zeta_{2}-\zeta_{2}\right)\right), \quad t \in[0,1]
$$


Die Beziehungen

und

$$
\begin{aligned}
& \alpha(z)=\arg \left[z^{\prime}(\zeta)\left(\zeta_{2}-\zeta_{1}\right)\left(z_{2}-z_{1}\right)^{-1}\right], \quad z \in \mathfrak{R}^{\prime}, \\
& |x(z)|=|\dot{z}(t)|^{-3}|\operatorname{Im}(\dot{z}(t) \ddot{z}(t))| \leqq\left|z^{\prime \prime}(\zeta)\right|\left|z^{\prime}(\zeta)\right|^{-2}=\left|\zeta^{\prime \prime}(z) / \zeta^{\prime}(z)\right|, \quad z \in \mathfrak{R}^{\prime},
\end{aligned}
$$

$$
\left|z_{1}-z_{2}\right| \leqq L=\int_{\mathscr{\Re}^{\prime}}|d z|=\left|\zeta_{2}-\zeta_{2}\right| \int_{0}^{1}\left|\zeta^{\prime}(z)\right|^{-1} d t
$$

liefern in Verbindung mit (1), (2),'(3) und (6) die Einschränkungen (20) bis (22)

Bemerkung 4: Unter Verwendung der Ungleichung (12) und der Abschätzung von $D\left(\mathfrak{\Re}^{\prime}\right)$ in 3.2. lassen sich leicht Kapazitätsabschätzungen für $\mathbb{\Re}^{\prime}\left(z_{1}, z_{2}\right)$ angeben, die asymptotisch scharf sind für $Q$ gegen 1 .

\section{LITERATUR}

[1] Bergman, S.: The kernel function and conformal mapping. New York 1950.

[2] Courant, R:: Dirichlet's principle, conformal mapping, and minimal surfaces. New York 1950 .

[3] Golosn, G. M.: Geometrische Funktionentheorie. Berlin 1957. (Ubers. a. d. Russ.)

[4] GrötzscH, H.: Uber ein Variationsproblem der konformen Abbildung. Ber: d. math.phys. Kl. d. Sächs. Akad. d. Wiss. zu Leipzig 82 (1930), $251-263$.

[5] JENkiNs, J. A.: Univalent functions and conformal mapping. Springer-Verlag: BerlinGöttingén-Heidelberg 1958.

[6] KIRSCH, S.: Ein verallgemeinerter transfiniter Durchmesser im Zusammenhang mit einer quasikonformen Normalabbildung. Complex Analysis: Banach Center Publications Voḷ. 11 (1982), $121-129$.

[7] KüHNA , R.: Quasikonforme Abbildungen und Extremalprobleme bei Feldern in inhomogenen Medien I. J. reine angew. Math. 231 (1968), $101-113$.

[8] -: Herleitung einiger Verzerrungseigenschaften konformer und allgemeinerer Abbildungen mit Hilfe des Argumentprinzips. Math. Nachr. 39 (1969), 249-275.

[9] - : Transfiniter Durchmesser, Kapazität und Tschebyschewsche Konstante in der euklidischen, hyperbolischen und elliptischen Geometrie. J. reine angew. Math. 234 (1969), $216-220$.

[10] - : Zur Methode der Randintegration bei quasikonformen Abbildungen. Ann. Polon. Math. 31 (1975), 269-289.

[11] - : Verzerrungsaussagen bei quasikonformen Abbildungen mit ortsabhängiger Dilatationsbeschränkung und ein Extremalprinzip der Elektrostatik in inhomogenen Medien. Comm. Math. Helvetici 53 (1978), 408-428.

[12] - : Gauss-Thomsonsches Prinzip minimaler Energie, verallgemeinerte transfinite Durchmesser und quasikonforme Abbildungen. In: Proc. Romanian-Finnish Seminar on Complex Analysis. Lect. Notes Math. 743 (1979), 140-164.

[13] - : Uber die Werte des Doppelverhältrisses bei quasikonformer Abbildung. Math. Nachr. $95(1980), 237-251$.

[14] Küxzr, H. P.: Quasikonforme Abbildungen. Springer-Verlag: Berlin-Göttingen-Heidelberg 1960.

[15] Кузьмин , Г. В.: Модули семейств кривых и квадратичные-дифференциалы. Труды ордена Ленина мат. ии.-та им. В. А. Стеклова СXXXIX, Ленинград 1980.

[16] Lento, O., und K. I. Virtanen: Quasikonforme Abbildungen. Springer-Verlag: BerlinHeidelberg - New York 1965.

[17] Parter, S. V.: On mappings of multiply connected domains by solutions of partial differential equations. Comm. pure appl. Math. 13 (1960), $167-182$. 
[18] PrRL, U.: Uber die geometrische Gestalt eines Extremalkontinuums aus der Theorie der konformen Abbildungen. Math. Nachr. 39 (1969), 297-312.

[19] Pfluakr, A.: Quasikonforme Abbildungen und logarithmische Kapazität. Ann. Inst. Fourier (Grenoble) 2 (1951), 69-70.

[20] REICr, E., and M. SchrFFr: Estimates for the transfinite diameter of a continuum. Math. Z. 85 (1964), $91-106$.

[21] SCHIFFER, M., and G. ScHOBER: Representation of fundamental solutions for generalized Cauchy-Riemann equations by quasiconformal mappings. Ann. Acad. Sci. Fenn., Ser. A, Vol..2(1976), 501-531.

[22] Szyвiak, A.: On some constants related to the generalized potentials. Ann. Polon. Màth. 6 (1959), 265-268.

Manuskripteingang: 06. 08. 1982; in revidierter Fassung: 01. 06. 1983

\section{VERFASSER}

Dr. STEGFried KirSCH

DDR-4020 Halle, Wilhelm-Pieck-Ring 81 